OPEN ACCESS

Edited by:

Yue Zhao,

University of Cologne, Germany

Reviewed by: Richard Chahwan,

University of Zurich, Switzerland Kui Ming Chan,

City University of Hong Kong, Hong Kong SAR, China

*Correspondence:

Quanyuan He hqyone@gmail.com

Specialty section:

This article was submitted to Molecular and Cellular Oncology, a section of the journal Frontiers in Cell and Developmental Biology

Received: 10 October 2021 Accepted: 16 December 2021 Published: 06 January 2022

Citation:

Zuo S, Yi Y, Wang C, Li X, Zhou M, Peng $Q$, Zhou J, Yang $Y$ and He $Q$ (2022) Extrachromosomal Circular

DNA (eccDNA): From Chaos

to Function.

Front. Cell Dev. Biol. 9:792555. doi: $10.3389 /$ fcell.2021.792555

\section{Extrachromosomal Circular DNA (eccDNA): From Chaos to Function}

\author{
Shanru Zuo ${ }^{1,2}$, Yihu $\mathrm{Yi}^{3}$, Chen Wang ${ }^{4}$, Xueguang $\mathrm{Li}^{2}$, Mingqing Zhou ${ }^{5}$, Qiyao Peng ${ }^{6,7}$, \\ Junhua Zhou ${ }^{2}$, Yide Yang $^{2}$ and Quanyuan $\mathrm{He}^{2 *}$
}

${ }^{1}$ Department of Pharmacy, The Third Xiangya Hospital, Central South University, Changsha, China, ${ }^{2}$ The Key Laboratory of Model Animals and Stem Cell Biology in Hunan Province, School of Medicine, Hunan Normal University, Changsha, China, ${ }^{3}$ Department of Orthopaedics, Wuhan Union Hospital, Wuhan, China, ${ }^{4}$ Department of Obstetrics and Gynecology, The Third Xiangya Hospital of Central South University, Changsha, China, ${ }^{5}$ Zhongshan Hospital Affiliated to Sun Yat-Sen University, Zhongshan People's Hospital, Zhongshan, China, ${ }^{6}$ Institute of Chinese Medicine, Hunan Academy of Traditional Chinese Medicine and Innovation Centre for Science and Technology, Hunan University of Chinese Medicine, Changsa, China, ${ }^{7}$ Chongqing Key Laboratory for Pharmaceutical Metabolism Research, College of Pharmacy, College of Traditional Chinese Medicine, Chongqing Medical University, Chongqing, China

Extrachromosomal circular DNA (eccDNA) is a type of double-stranded circular DNA that is derived and free from chromosomes. It has a strong heterogeneity in sequence, length, and origin and has been identified in both normal and cancer cells. Although many studies suggested its potential roles in various physiological and pathological procedures including aging, telomere and rDNA maintenance, drug resistance, and tumorigenesis, the functional relevance of eccDNA remains to be elucidated. Recently, due to technological advancements, accumulated evidence highlighted that eccDNA plays an important role in cancers by regulating the expression of oncogenes, chromosome accessibility, genome replication, immune response, and cellular communications. Here, we review the features, biogenesis, physiological functions, potential functions in cancer, and research methods of eccDNAs with a focus on some open problems in the field and provide a perspective on how eccDNAs evolve specific functions out of the chaos in cells.

Keywords: eccDNA, circulome, biogenesis, cancer, biomarker

\section{INTRODUCTION}

EccDNA refers to a type of double-stranded circular DNA that is derived and free from chromosomes. In 1965, Alix Bassel and Yasuo Hoota first observed eccDNA in boar sperm using the electron microscope (Hotta and Bassel, 1965). From there, many efforts have been taken to figure out the features and functions of eccDNAs (Figure 1). It is clear now that eccDNA is ubiquitous in eukaryotic cells and has been identified in yeasts, plants, Oxytricha, Xenopus, pigeons, and human cells. (Cohen and Méchali, 2002; Møller et al., 2015; Hull et al., 2019; Yerlici et al., 2019; Molin et al., 2020a, Molin et al., 2020b; Møller et al., 2020; Sin et al., 2021; Wang K et al., 2021; Zhu et al., 2021). It can be derived from everywhere in a genome with sizes ranging from hundreds of base pairs (bp) to several mega bases $(\mathrm{Mb})$. According to the size and origin, eccDNAs can be categorized into mitochondria DNAs (mtDNAs), episomes, double minutes (DMs) (100kb 3 Mb), telomeric circle (t-circles), small polydispersed circular DNA (spcDNA) (100bp 10 kb), and microDNA (100-400 bp) (Wang $M$ et al., 2021).Some studies revealed that episomes can be polymerized into DMs in cancer cells suggesting the possibility that one type of eccDNA can be transformed to others by polymerization or fragmentation with subsequent recircularization (Wahl et al., 1984; Carroll et al., 1988). 


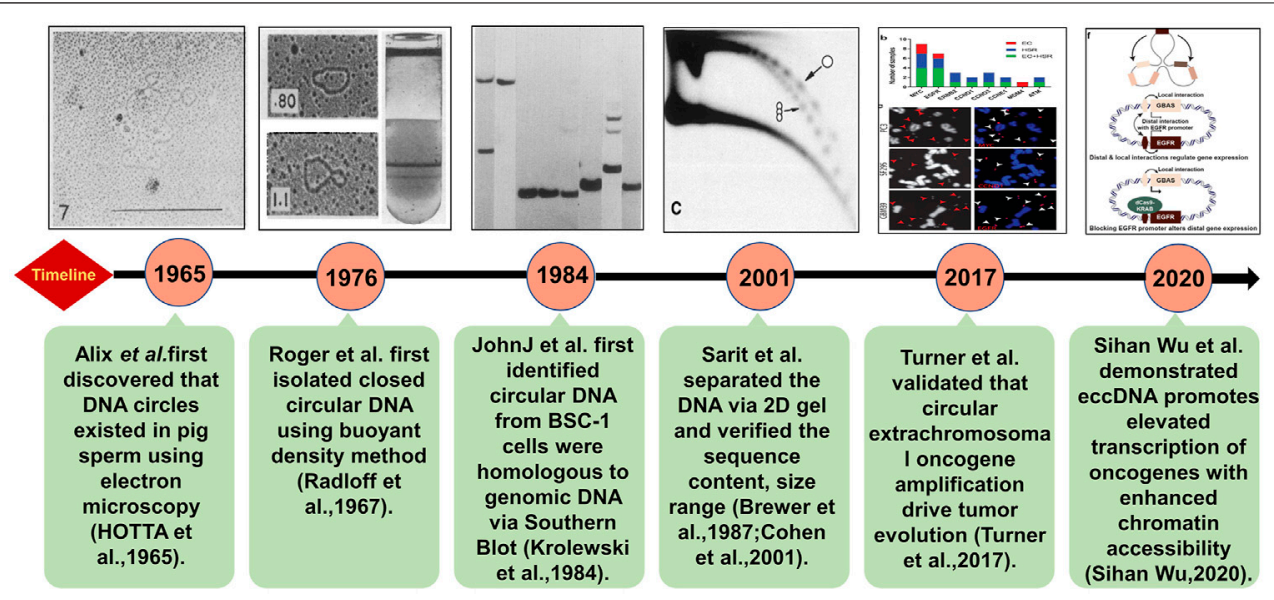

FIGURE 1 | Brief discovery history of eccDNA. The technologies and key discoveries were illustrated following the timeline of the milestone studies. The detailed descriptions are as following: in 1965, Alix Bassel and Yasuo Hoota first observed eccDNAs in boar sperm under the electron microscope (Hotta and Bassel, 1965). The Roger's group extracted eccDNA in HeLa cells by CsCl gradient purification in 1976 (Radloff et al., 1967). In 1984, Krolewski found that eccDNA molecules were homologous to genomic DNA (Krolewski et al., 1984) using Southern blot indicating that eccDNAs may be derived from chromosomes. Neutral-neutral twodimensional (2D) gel electrophoresis combined with standard procedures of Southern blots and hydridization verified useful for characterizing eccDNA including its size range and sequence content, and it can prove eccDNA organized as discrete multimers (Brewer and Fangman, 1987; Cohen and Mechali, 2001). With rapid advances in high-throughput DNA sequencing, more eccDNAs have been identified and quantified on a global level (Turner et al., 2017). Furthermore, the architecture of eccDNA can be directly observed by super resolution (SR) confocal microscopy (Sinan Wu, 2020).
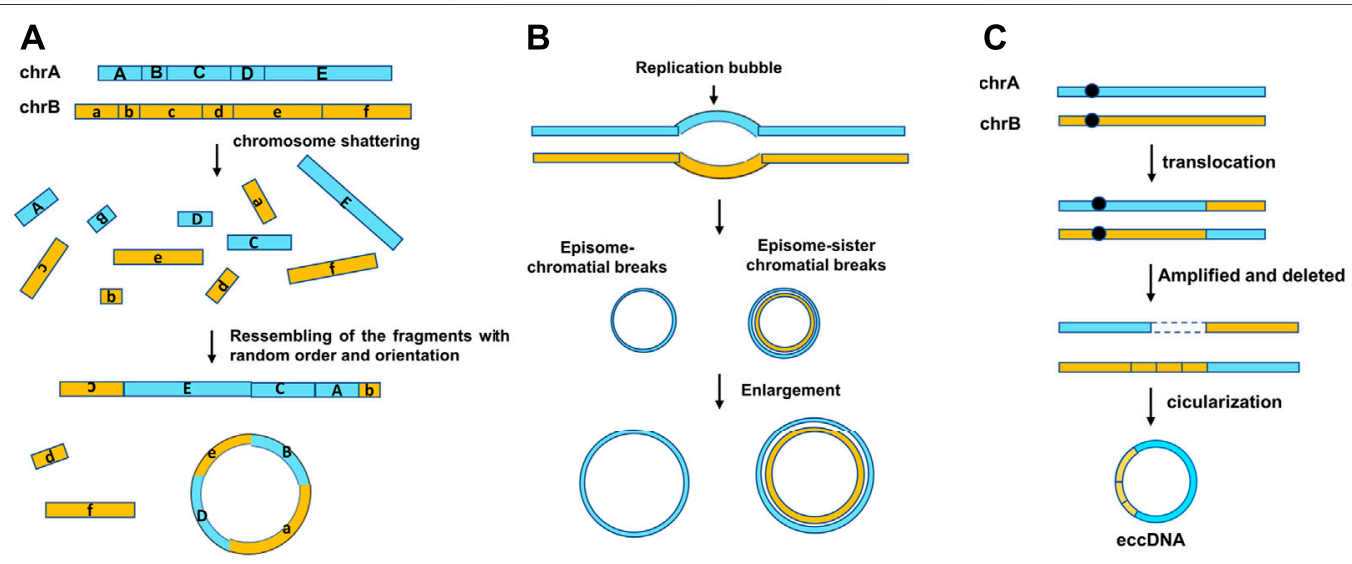

FIGURE 2 | Three mechanisms of eccDNA biogenesis. (A) "Chromothripsis" model, chromothripsis is the generation of DNA double-strand breaks. Fragments are joined together in a random order and orientation by DNA repair machineries. The new chromosomes may contain complex structural rearrangements and structural variants. (B) Episome model, DNA bidirectionally replicates leading to two replication forks, and the region between them is the replication bubble. The replication fork collapse when the error in replication take place, and then, the replication bubbles drop and form shape of an episome. They can enlarge to form eccDNAs by replication and recombination. (C) Translocation-excision-deletion-amplification model. Gene translocation occurs near the chromosome. The fragment next to the translocation positions can be amplified, deleted, and circularized, generating the genesis of eccDNA.

As the counts, compositions, origin, and expression patterns of eccDNA are dramatically dynamic and diverse in cells and tissues, characterizing the functions and underline mechanisms of eccDNAs are extremely challenging (Turner et al., 2017). Many fundamental open questions about eccDNAs remained unanswered. For example, is eccDNA a byproduct or essential player of cellular procedures? Do the sequences of eccDNAs matter for their functions? What is the role of eccDNAs in cancer initiation and progression? et al. Some excellent reviews (Cao et al., 2021; Ling et al., 2021; Wang $T$ et al., 2021) have summarized the characteristics and functions of eccDNA. Here, we review the last progress in the field and try to find the clues to answer the above questions.

\section{THE BIOGENESIS OF ECCDNA}

Several models of the eccDNA biogenesis have been proposed: 1) The chromothripsis model (Figure 2A): Chromothripsis is the catastrophic shattering of a chromosome followed by massive 
genomic rearrangement in a random order, leading to complex genomic structural rearrangements in confined genomic regions. As the "shattering" procedure results in clustered DNA doublestrand breaks (DSBs), following by DNA repairing or aberrant DNA replication, and which make it a perfect circumstance to generate eccDNA (Nazaryan-Petersen et al., 2020); in 2021, Wang's team (Wang $Y$ et al., 2021) reported that apoptosis can promote eccDNA generation in human and mouse cells. It is not surprising; apoptosis, just like chromothripsis, can induce DNA fragmentation and may provide massive DNA fragments for eccDNA formation; 2) Episome model (Figure 2B). Replication fork stalling may result in replication fork collapse, and the replication bubble subsequently falls off the chromosome, transforms into an extrachromosomal circular molecule named as episome (Gu et al., 2020); 3) Translocation-excision-deletion-amplification model (Figure 2C) (Boon et al., 2001; Van Roy et al., 2006). In this model, DNA rearrangements take place close to translocation sites on the chromosome. The fragment in proximity to the translocation breakpoints can be amplified, deleted, and circularized, resulting in the accumulation of eccDNAs.

The above studies indicated that the DNA damage repairing pathways including homologous recombination (HR) or nonhomologous end-joining (NHEJ) and microhomologymediated end-joining (MMEJ) are involved in the formation of microDNAs (Dillon et al., 2015; Paulsen et al., 2021). The cells lack 53BP1 and XRCC4 promoting the canonical-NHEJ (c-NHEJ), resulting in more microDNA. On the contrary, the deficiency of NBS1, POLQ, RAD54, MLH1, MSH2, MSH3, FAN1, and NBS results in the decrease of microDNA (Paulsen et al., 2021). The short, reverted repeats at both ends of junction sites provide perfect places to initialize homologous recombination (HR) during eccDNA formation. Furthermore, some key players in NHEJ and MMEJ pathways, such as DNA ligases IV and III, were found to contribute to the eccDNA generation (Cohen et al., 2006; Wang K et al., 2021). Additionally, DNA replication and transcription were also proposed as the mechanisms underlying the formation of eccDNAs. For instance, the most transcribed protein-coding gene in the muscle, titin (TTN), has the greatest amount of eccDNAs (Møller et al., 2018). On the contrary, noncoding gene areas show a lower correlation with the eccDNA frequency (Dillon et al., 2015; Møller et al., 2018).

It is widely accepted that eccDNA origination is not restricted to a particular locus in the genome (Møller et al., 2020; Wang T et al., 2021). However, the pattern of eccDNA distribution is still controversial. Some studies reported that the vast majority of eccDNA originates from repetitive elements (Motejlek et al., 1993; Cohen and Mechali, 2001; Moller et al., 2015); others suggested that they prefer to reside in some hotspots such as UTRs of genes, GC islands, and transcriptionally activated chromatins (Shibata et al., 2012; Mehanna et al., 2017). A recent study supported an even distribution model in drug-induced apoptosis cells (Wang Y et al., 2021). Although the driving force of these patterns is still unknown, it is reasonable to speculate that eccDNA origination may be determined by the mechanisms of eccDNA generation under different cellular conditions (Figure 3).

\section{THE AMPLIFICATION OF ECCDNA}

Most of eccDNA will be degraded rapidly except the ones who can amplify themselves. However, what are the mechanisms of eccDNA amplification is controversial. Some studies suggested that eccDNA amplification relies on DNA replication and mitosis (Baker and Grant, 2007; Herrup and Yang, 2007); others have found that the eccDNA level increases when the ongoing replication is blocked by inhibitors (Sunnerhagen et al., 1986) or even in the absence of any DNA replication (Cohen et al., 2009). However, it is not clear whether these eccDNA levels increasing are a result from amplification or higher levels of biogenesis. We speculate that eccDNAs can be amplified in a way independent of mitosis because many of them don't have replication origins which are required by regular DNA replication.

\section{REGULATORY MECHANISMS OF ECCDNA}

Accumulated evidence suggested that eccDNA may regulate many cellular procedures (see the function sections below) through distinct mechanisms (Figure 3): 1) Biogenesis of eccDNAs containing whole gene may lead to a gain in the gene copy number and enhance the transcription of the gene (Turner et al., 2017); 2) Forming R-loop by hybridization with mRNA to regulate its translational efficiency (Wiedemann et al., 2016); 3) Titrating the components of the replication or transcription machinery and result in an inability to replicate or transcribe genomic DNA, which eventually induces cell growth arrest and death; (Paulsen et al., 2019; Zhu, et al., 2021); 4) Acting as trans-acting factors (such as super enhancer) to regulate the gene expression by regulating the epigenetic statue and/or accessibility of the targeting gene (Wu et al., 2019); 5) Function as a cytokine to stimulate immune responses or mediate the intercellular communication (Poirier et al., 2018); 6. Genetic rearrangements by the reinsertion of eccDNA into the genome DNA (Pavri, 2017); 7. Protecting the telomeres in ALT cells (McEachern, 2000; Cech, 2004).

\section{PHYSIOLOGICAL FUNCTIONS OF ECCDNA}

It is already known that eccDNA plays an important role in a variety of physiological procedures including the following: 1) Stress resistance and evolution. In plants, eccDNA plays a role to amplify and transmit the herbicide resistance gene in crop weeds which result in rapid glyphosate resistance and adaptive evolution (Koo et al., 2018). Furthermore, eccDNA was identified in the C.elegans germ line and may serve as genetic materials to be inherited by the offspring (Shoura et al., 2017); 2) Functional enhancement. In humans, the most transcribed protein-coding gene in the muscle, titin (TTN), has the greatest amount of eccDNAs (Møller et al., 2018), and suggesting eccDNA may help host cell fulfill their function. 3) Aging. EccDNA has been found accumulated in old cells and inducing aging in yeast (Hull et al., 2019). It was also amplified during aging in senescence-resistant SAM-R mice and associated with premature aging and shorter lifespan (Yamagishi et al., 1985). Accumulated results highlighted 


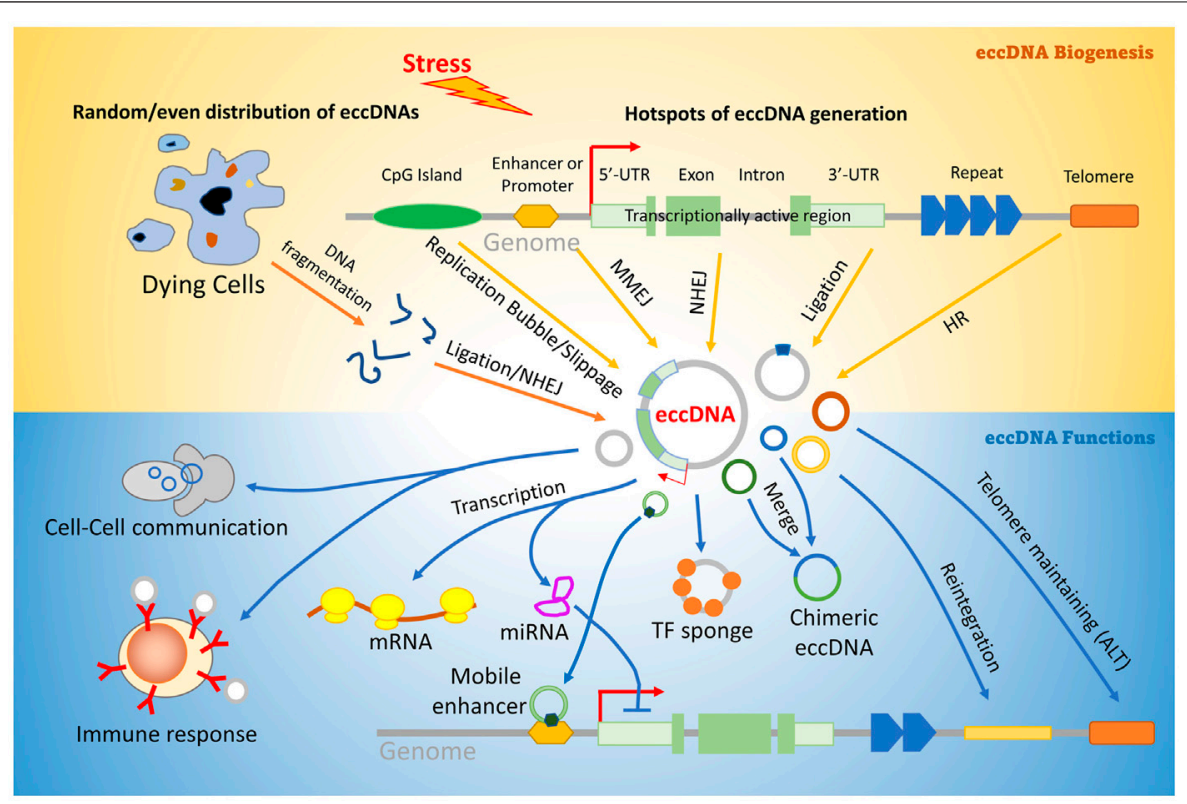

FIGURE 3 | Pathways involved in eccDNA biogenesis and function. Stress may induce aberrant DNA replication, transcription, or even genomic DNA broken into random fragments, and generate linear DNA fragments. Some of them are ligated and circularized by DNA damage repair pathways including HR (homologous recombination), NHEJ (Non-homologous end joining), Ligation by DNA ligases IV and III, and MMEJ (microhomology mediated end-joining). EccDNAs can regulate many downstream biological procedures including transcription, telomere protection, gene translocation, immune response, and cell-cell communication. Description about details can be found in the text.

the proposed role of eccDNAs in age-related CNS diseases, but direct evidence is still absent (Shibata et al., 2012); 4) Genome stability maintenance. Certain types of eccDNAs were originated from telomeres ( $\mathrm{t}$-circles) and centromeres (major satellite repeats), that are fundamental structures to maintain genome integrity. Especially, t-circle is essential to maintain telomeres in ATL cells (Neumann et al., 2013); 5) Immune response. As not protected by chromatin, eccDNA may become an endogenous antigen to active autoimmune cells (Collins et al., 2004). Recently, the role of eccDNA in triggering innate immune response was uncovered (Wang M et al., 2021). Surprisingly, the study demonstrated that it is the circular nature but not the sequence of eccDNA critical to the immune response activation; 6) Cell-to-cell communications. The high stability, high mobility, and self-replication ability of eccDNA make it a perfect messenger to transduce and amplify signals among cells. Recent studies confirmed that eccDNA (especially microDNA) can be emitted and sensed by human and mouse cells efficiently in vitro (Wang $\mathrm{T}$ et al., 2021). In addition, the eccDNAs released by normal and tumor cells have been detected in the peripheral blood (Møller et al., 2018). These data suggested a potential role of eccDNA contributing to both local and long-range signal transduction between cells.

\section{FUNCTIONS OF ECCDNA IN CANCER}

EcDNA refers to large eccDNA exclusively detected in tumors. Furthermore, DM is a specific type of ecDNA, which usually contains intact oncogenes and/or drug-resistance genes, which has been detected in most (182/200) kinds of human tumors (Fan et al., 2011). EcDNA, as the vehicles for oncogene and drugresistance genes, enables them to be rapidly amplified, and lead to overexpression consequently (Turner et al., 2017). For instance, oncogenes EGFR and c-MYC were found in ecDNA and amplified in human cancer tissues than normal tissues (L'Abbate et al., 2014). In tumor cells from a SCLC (small cell lung cancer) patient who received methotrexate (MTX), a large quantity of DMs containing dihydrofolate reductase (DHFR), a drug-resistance gene, was amplified and overexpressed (Curt et al., 1983). Lacking a high-order chromatin structure, suppressing histone modifications, and insulator shackle make ecDNAs more accessible than their genome counterparts which may facilitate promoter-enhancer interactions, transcription initialization, and achieving additional expression (Morton et al., 2019). Furthermore, recent studies also suggested that ecDNA/DMs can function as mobile super-enhancers to enhance targeting genes transcription in genome-wide (Y. Zhu, et al., 2021). Additionally, the unbalanced segregation of ecDNA during mitotic divisions provides an additional layer of tumor heterogeneity which ultimately cause tumor adaptation to the microenvironment stresses and various therapies such as radiotherapy and chemotherapy. This may be the reason that tumors with ecDNA are frequently more aggressive, related to poorer survival outcomes (Bruckert et al., 2000).

The role of smaller eccDNAs (e.g., microDNAs) in cancer biology remains controversial. As they usually are too small to contain the full length of the gene (except for miRNA genes), 
whether its transcriptional products have any effects on cancer progression is unknown. However, they may be important for molecular sponging. As often originated from the $5^{\prime}$-UTRs of its parental gene, microDNA may provide additional binding sites for related transcription factors and therefore, acting as a sponge for transcription factors to control the gene expression and transcription homeostasis indirectly (Reon and Dutta, 2016; Paulsen et al., 2018). Additionally, Wang et al. reported that the microDNAs released from dying cells can dramatically induce type I interferon expression and innate immunostimulatory response. It is suspected that drug treatment on cancer cells may induce cell apoptosis with eccDNA generation and subsequent change in the tumor microenvironment dramatically. This work highlighted the potential role of microDNA in drug resistance of cancer cells and shed new light on cancer immunotherapy (Wang Y et al., 2021).

\section{APPLICATIONS OF ECCDNA IN MEDICINE}

The entire set of circular DNA (Circulome) varies as a function of tissue type and health and disease conditions and can be used as a fingerprint of disease. Extracellular free eccDNAs are more stable than linear cell-free DNAs (cfDNAs) and have been detected in cancer tissues and peripheral blood of cancer patients (Kumar et al., 2017), suggesting the potential applying them as a novel type of biomarkers in liquid biopsy for the early detection of diseases, the monitoring of drug treatment response, and cancer survival.

Although many efforts have been made, the application of eccDNA in medicine is still at an early stage. In the mammalian repertoire of eccDNA, microDNAs may be the most promising biomarkers because they have smaller size, higher mobility, and higher abundance (account for up to $84 \%$ of the entire circulome) (Shibata et al., 2012; Dillon et al., 2015) than others. Furthermore, it exhibited lineage-specific or cell type-specific patterns in human cells (Hotta and Bassel, 1965; Dillon et al., 2015). However, the functions of microDNA are largely unknown, and very few of them are shared by patients, which raise the questions whether the sequence is a matter for them or not. Large cohort studies are needed to identify clinic-relevant microDNA patterns. Another potential eccDNA biomarker is mtDNA. Some studies have reported that the mtDNA copy number variation was correlated with cancer (Sozzi et al., 2003). For example, a high mtDNA copy number in peripheral blood leukocytes (PBLs) was associated with the increased risk of prostate cancer (PCa) and high tumor burden in PCa patients (Zhou et al., 2014). And paradoxically, another report indicated that low mtDNA abundance in PBLs correlated with aggressive PCa at diagnosis (Tu et al., 2015). Similarly, the mtDNA copy number was significantly lower in PBLs of patients with endometrial cancer than normal controls (Sun et al., 2016). As these associations between mtDNA amount and cancer risk are inconsistent and the underlying mechanisms are still unknown, huge gap needs to be filled before the clinical application of mtDNAs. Moreover, it remains unclear about eccDNAs' presence in other bodily fluids such as saliva and urine. It is worthwhile to check the existence of eccDNAs in other bodily fluids.

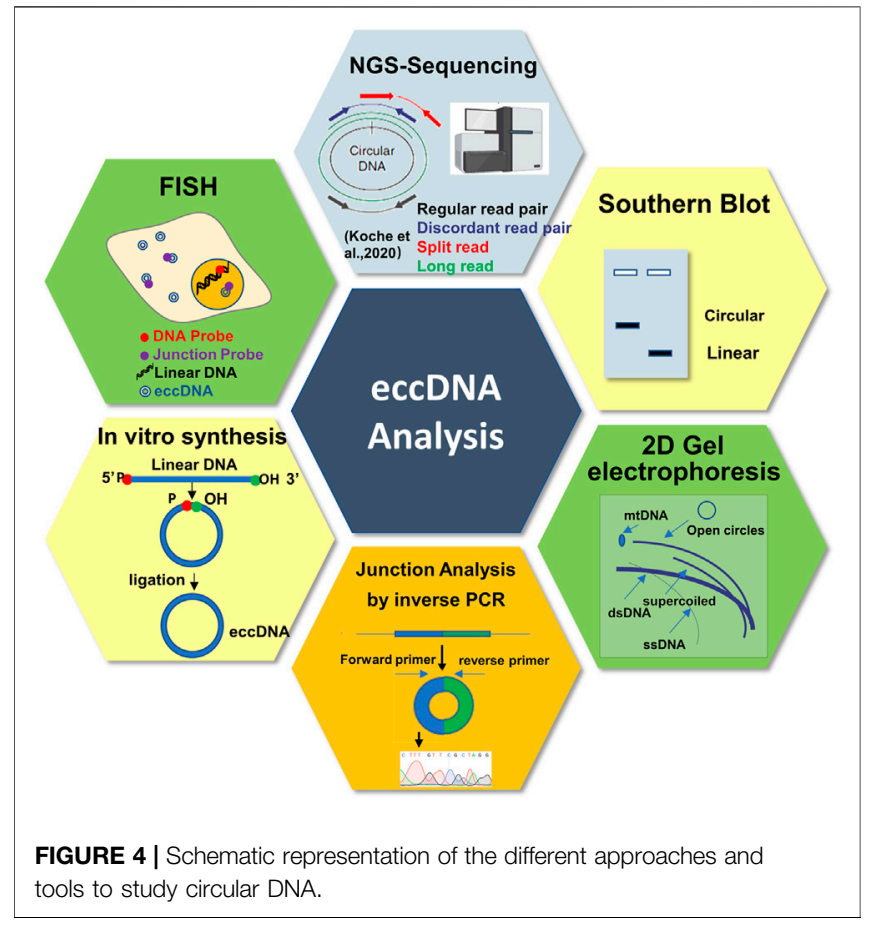

\section{ADVANCE OF ECCDNA RESEARCH METHODS}

Many conventional methods were used to characterize the sequence, copy number, subcellular localization, and biological functions of eccDNAs (Figure 4), and which was reviewed by elsewhere (Wang T et al., 2021). However, these methods usually provide little information about eccDNA sequences and cannot provide the global view for the whole circulome. Currently, the next/third generation sequencing technologies (such as long-read sequencing (Nanopore and PacBio SMRT sequencing) and single cell sequencing (SC-Seq)) and the improved methods (such as Circle-seq, 4C-seq, PLAC-seq, and ATAC-seq) provide us revolutionary tools to investigate the circulome from various perspectives and with single base-pair and single-cell resolutions (Turner et al., 2017; Prada-Luengo et al., 2019; Rajkumar et al., 2019; Kumar et al., 2020; Mehta et al., 2020; Sin et al., 2020). Especially, the long-read sequencing coupled with the PCR-free eccDNA purification technology present a promising solution to overcome the limitation of short-read sequencing in detecting big eccDNAs and provide unbiased circulomics profiles (Wang M et al., 2021). Furthermore, we speculated that the SC-Seq may be the next dominant technology to decode the rule of eccDNA segregation to rebuild the evolutionary history of the tumor (van den Bos et al., 2018). Additionally, recently, many bioinformatics analysis tools and database for eccDNAs have been proposed (Rajkumar et al., 2019; Wu et al., 2019), which facilitate the rapid development of eccDNA research significantly. However, taking the extreme complexity of the circulome into consideration, the data about eccDNA are still very limited; intensive studies need to be carried out to accumulate and annotate eccDNAs in the future. 


\section{PERSPECTIVE: FROM CHAOS TO FUNCTION}

For most of eccDNAs, the story may begin with a chaos result from certain internal and external stresses. The stress induces aberrant transcription, DNA replication, and recombination leading to the release of DNA fragments from some hotspots which were consequently recognized and "repaired" by DNA damage repairing mechanisms to form a circulated DNA. In dying or apoptosis cell, global genome DNA fragmentation generate a lot of DNA fragments in a random fashion; some of them were also "rescued" by DNA damage repairing mechanisms to form eccDNAs. We speculate that the former mechanism may majorly contribute to the formation of relatively longer eccDNAs (episomes and DMs), and the latter one is for shorter eccDNAs (spcDNA and microDNA). Most of the eccDNAs will be degraded rapidly, and only the ones who have the ability to amplify themselves and provide adaptive advantage to cells can survive. During the procedure of evolutionary selection, eccDNAs may migrate through cells to search for a good host. They can merge with others to create a new one and novel functions which may provide further advantages of the survival of themselves and hosts. Some eccDNAs may be even more "lucky" to get the chance to integrate into genomic DNA, which eventually fixes themselves in the cell's genome. In tumors, this story may happen everywhere and all the time. EccDNAs help cancer cells develop drug resistance and escape from immune attack. Furthermore, it may also be possible that the eccDNAs originated from the cancer cell can transform target cells remotely through circulation and mediate the metastasis. In germ line, eccDNAs may serve as the secondary genetic materials and were passed to progeny and create additional genetic diversity in population. For somatic cells, too much random eccDNAs may be a burden. However, highly specific functional eccDNA may be a big help for them to fulfill their functions especially under stress conditions (Møller et al., 2018).

\section{CONCLUSION}

The discovery of eccDNA may reshape our current understanding of the heredity, evolution, and diseases.

\section{REFERENCES}

Baker, S. P., and Grant, P. A. (2007). The SAGA Continues: Expanding the Cellular Role of a Transcriptional Co-Activator Complex. Oncogene. 26 (37), 5329-5340. doi:10.1038/sj.onc.1210603

Boon, K., Caron, H. N., van Asperen, R., Valentijn, L., Hermus, M.-C., van Sluis, P., et al. (2001). N-myc Enhances the Expression of a Large Set of Genes Functioning in Ribosome Biogenesis and Protein Synthesis. Embo J. 20 (6), 1383-1393. doi:10.1093/emboj/20.6.1383

Brewer, B. J., and Fangman, W. L. (1987). The Localization of Replication Origins on ARS Plasmids in S. Cerevisiae. Cell. 51 (3), 463-471. doi:10.1016/00928674(87)90642-8

Bruckert, P., Kappler, R., Scherthan, H., Link, H., Hagmann, F.-G., and Zankl, H. (2000). Double Minutes and C-MYC Amplification in Acute Myelogenous
EccDNA, as a new kind of genetic material, is mobile, flexible, functionable, tremendous diverse, and everywhere (Wang $\mathrm{T}$ et al., 2021). It lets all the cells different and makes quick adaptation and evolution possible. The biological significance of eccDNAs may be heavily underestimated. The eccDNA study is still in its infancy.

Future studies need to focus on 1) Figuring out the chromatin structural features and sequence characteristics of eccDNA on the circulome level; 2) Discovering key regulators and interaction partners of eccDNAs; 3) Exploring the eccDNA segregation procedure at the single cell level to understanding cancer development; 4) Applying it as a liquid biopsy biomarker to improve disease diagnosis, prognosis, and treatment selection; 5) Developing more convenience and sensitive detection methods and advanced tools to support eccDNA research. All these works will deepen our understanding of the nature of eccDNAs and open up new opportunities for cancer diagnosis and therapy.

\section{AUTHOR CONTRIBUTIONS}

SZ and $\mathrm{QH}$ conceived the present idea and researched the background of the study. YY, CW, XL, QP, MZ, YY, and JZ prepared the figures. SZ, YY, CW, and QH wrote the manuscript. $\mathrm{QH}$ revised the manuscript. All the authors read and approved the final manuscript.

\section{FUNDING}

This work was supported by the National Natural Science Foundation of China (31771445), the Major Scientific and Technological Project for Collaborative Prevention and Control of Birth Defect in Hunan Province (2019SK1012), and the High-Level Talent Program in Hunan Province (2019RS1035).

\section{ACKNOWLEDGMENTS}

We acknowledge and appreciate our colleagues for their valuable suggestions and technical assistance for this study.

Leukemia. Cancer Genet. Cytogenet. 120 (1), 73-79. doi:10.1016/S01654608(99)00235-6

Cao, X., Wang, S., Ge, L., Zhang, W., Huang, J., and Sun, W. (2021). Extrachromosomal Circular DNA: Category, Biogenesis, Recognition, and Functions. Front. Vet. Sci. 8, 693641. doi:10.3389/fvets.2021.693641

Carroll, S. M., DeRose, M. L., Gaudray, P., Moore, C. M., NeedhamVandevanter, D. R., Von Hoff, D. D., et al. (1988). Double Minute Chromosomes Can Be Produced from Precursors Derived from a Chromosomal Deletion. Mol. Cell. Biol. 8 (4), 1525-1533. doi:10.1128/ mcb.8.4.1525-1533.1988

Cech, T. R. (2004). Beginning to Understand the End of the Chromosome. Cell. 116 (2), 273-279. doi:10.1016/s0092-8674(04)00038-8

Cohen, S., and Mechali, M. (2001). A Novel Cell-free System Reveals a Mechanism of Circular DNA Formation from Tandem Repeats. Nucleic Acids Res. 29 (12), 2542-2548. doi:10.1093/nar/29.12.2542 
Cohen, S., and Méchali, M. (2002). Formation of Extrachromosomal Circles from Telomeric DNA in Xenopus laevis. EMBO Rep. 3 (12), 1168-1174. doi:10.1093/ embo-reports/kvf240

Cohen, Z., Bacharach, E., and Lavi, S. (2006). Mouse Major Satellite DNA Is Prone to eccDNA Formation via DNA Ligase IV-dependent Pathway. Oncogene. 25 (33), 4515-4524. doi:10.1038/sj.onc. 1209485

Cohen, Z., Lavi, S., and Sullivan, B. A. (2009). Replication Independent Formation of Extrachromosomal Circular DNA in Mammalian Cell-Free System. PLoS One. 4 (7), e6126. doi:10.1371/journal.pone.0006126

Collins, L. V., Hajizadeh, S., Holme, E., Jonsson, I.-M., Tarkowski, A., Sahlgrenska, A., et al. (2004). Endogenously Oxidized Mitochondrial DNA Induces In Vivo and In Vitro Inflammatory Responses. J. Leukoc. Biol. 75 (6), 995-1000. doi:10.1189/jlb.0703328

Curt, G. A., Carney, D. N., Cowan, K. H., Jolivet, J., Bailey, B. D., Drake, J. C., et al. (1983). Unstable Methotrexate Resistance in Human Small-Cell Carcinoma Associated with Double Minute Chromosomes. N. Engl. J. Med. 308 (4), 199-202. doi:10.1056/NEJM198301273080406

Dillon, L. W., Kumar, P., Shibata, Y., Wang, Y.-H., Willcox, S., Griffith, J. D., et al. (2015). Production of Extrachromosomal MicroDNAs Is Linked to Mismatch Repair Pathways and Transcriptional Activity. Cell Rep. 11 (11), 1749-1759. doi:10.1016/j.celrep.2015.05.020

Fan, Y., Mao, R., Lv, H., Xu, J., Yan, L., Liu, Y., et al. (2011). Frequency of Double Minute Chromosomes and Combined Cytogenetic Abnormalities and Their Characteristics. J. Appl. Genet. 52 (1), 53-59. doi:10.1007/s13353-010-0007-z

Gu, X., Yu, J., Chai, P., Ge, S., and Fan, X. (2020). Novel Insights into Extrachromosomal DNA: Redefining the Onco-Drivers of Tumor Progression. J. Exp. Clin. Cancer Res. 39 (1), 215. doi:10.1186/s13046-02001726-4

Herrup, K., and Yang, Y. (2007). Cell Cycle Regulation in the Postmitotic Neuron: Oxymoron or New Biology? Nat. Rev. Neurosci. 8 (5), 368-378. doi:10.1038/ nrn2124

Hotta, Y., and Bassel, A. (1965). Molecular Size and Circularity of Dna in Cells of Mammals and Higher Plants. Proc. Natl. Acad. Sci. 53, 356-362. doi:10.1073/ pnas.53.2.356

Hull, R. M., King, M., Pizza, G., Krueger, F., Vergara, X., and Houseley, J. (2019). Transcription-induced Formation of Extrachromosomal DNA during Yeast Ageing. Plos Biol. 17 (12), e3000471. doi:10.1371/journal.pbio.3000471

Koo, D.-H., Molin, W. T., Saski, C. A., Jiang, J., Putta, K., Jugulam, M., et al. (2018). Extrachromosomal Circular DNA-Based Amplification and Transmission of Herbicide Resistance in Crop weedAmaranthus Palmeri. Proc. Natl. Acad. Sci. USA. 115 (13), 3332-3337. doi:10.1073/pnas.1719354115

Krolewski, J. J., Schindler, C. W., and Rush, M. G. (1984). Structure of Extrachromosomal Circular DNAs Containing Both the Alu Family of Dispersed Repetitive Sequences and Other Regions of Chromosomal DNA. J. Mol. Biol. 174 (1), 41-54. doi:10.1016/0022-2836(84)90364-4

Kumar, P., Dillon, L. W., Shibata, Y., Jazaeri, A. A., Jones, D. R., and Dutta, A. (2017). Normal and Cancerous Tissues Release Extrachromosomal Circular DNA (eccDNA) Into the Circulation. Mol. Cancer Res. 15 (9), 1197-1205. doi:10.1158/1541-7786.MCR-17-0095

Kumar, P., Kiran, S., Saha, S., Su, Z., Paulsen, T., Chatrath, A., et al. (2020). ATACseq Identifies Thousands of Extrachromosomal Circular DNA in Cancer and Cell Lines. Sci. Adv. 6 (20), a2489. doi:10.1126/sciadv.aba2489

L'Abbate, A., Macchia, G., D'Addabbo, P., Lonoce, A., Tolomeo, D., Trombetta, D., et al. (2014). Genomic Organization and Evolution of Double Minutes/ Homogeneously Staining Regions withMYCamplification in Human Cancer. Nucleic Acids Res. 42 (14), 9131-9145. doi:10.1093/nar/gku590

Ling, X., Han, Y., Meng, J., Zhong, B., Chen, J., Zhang, H., et al. (2021). Small Extrachromosomal Circular DNA (eccDNA): Major Functions in Evolution and Cancer. Mol. Cancer 20 (1), 113. doi:10.1186/s12943-021-01413-8

McEachern, M. J., Krauskopf, A., and Blackburn, E. H. (2000). Telomeres and Their Control. Annu. Rev. Genet. 34, 331-358. doi:10.1146/ annurev.genet.34.1.331

Mehanna, P., Gagné, V., Lajoie, M., Spinella, J.-F., St-Onge, P., Sinnett, D., et al. (2017). Characterization of the microDNA Through the Response to Chemotherapeutics in Lymphoblastoid Cell Lines. PLoS One. 12 (9), e0184365. doi:10.1371/journal.pone.0184365

Mehta, D., Cornet, L., Hirsch-Hoffmann, M., Zaidi, S. S.-e. -A., and Vanderschuren, H. (2020). Full-length Sequencing of Circular DNA Viruses and Extrachromosomal Circular DNA Using CIDER-Seq. Nat. Protoc. 15 (5), 1673-1689. doi:10.1038/s41596-020-0301-0

Molin, W. T., Yaguchi, A., Blenner, M., and Saski, C. A. (2020a). Autonomous Replication Sequences from the Amaranthus Palmeri eccDNA Replicon Enable Replication in Yeast. BMC Res. Notes. 13 (1), 330. doi:10.1186/s13104-02005169-0

Molin, W. T., Yaguchi, A., Blenner, M., and Saski, C. A. (2020b). The EccDNA Replicon: A Heritable, Extranuclear Vehicle That Enables Gene Amplification and Glyphosate Resistance in Amaranthus Palmeri. Plant Cell. 32 (7), 2132-2140. doi:10.1105/tpc.20.00099

Møller, H. D., Larsen, C. E., Parsons, L., Hansen, A. J., Regenberg, B., and Mourier, T. (2016). Formation of Extrachromosomal Circular DNA from Long Terminal Repeats of Retrotransposons in Saccharomyces cerevisiae. G3 (Bethesda). 6 (2), 453-462. doi:10.1534/g3.115.025858

Møller, H. D., Mohiyuddin, M., Prada-Luengo, I., Sailani, M. R., Halling, J. F., Plomgaard, P., et al. (2018). Circular DNA Elements of Chromosomal Origin Are Common in Healthy Human Somatic Tissue. Nat. Commun. 9 (1), 1069. doi:10.1038/s41467-018-03369-8

Møller, H. D., Parsons, L., Jørgensen, T. S., Botstein, D., and Regenberg, B. (2015). Extrachromosomal Circular DNA Is Common in Yeast. Proc. Natl. Acad. Sci. USA. 112 (24), E3114-E3122. doi:10.1073/pnas.1508825112

Møller, H. D., Ramos-Madrigal, J., Prada-Luengo, I., Gilbert, M. T. P., and Regenberg, B. (2020). Near-Random Distribution of Chromosome-Derived Circular DNA in the Condensed Genome of Pigeons and the Larger, More Repeat-Rich Human Genome. Genome Biol. Evol. 12 (2), 3762-3777. doi:10.1093/gbe/evz281

Morton, A. R., Dogan-Artun, N., Faber, Z. J., MacLeod, G., Bartels, C. F., Piazza, M. S., et al. (2019). Functional Enhancers Shape Extrachromosomal Oncogene Amplifications. Cell. 179 (6), 1330-1341. doi:10.1016/j.cell.2019.10.039

Motejlek, K., Schindler, D., Assum, G., and Krone, W. (1993). Increased Amount and Contour Length Distribution of Small Polydisperse Circular DNA (spcDNA) in Fanconi Anemia. Mutat. Research/DNA Repair. 293 (3), 205-214. doi:10.1016/0921-8777(93)90071-n

Nazaryan-Petersen, L., Bjerregaard, V. A., Nielsen, F. C., Tommerup, N., and Tümer, Z. (2020). Chromothripsis and DNA Repair Disorders. J. Clin. Med. 9 (3), 613. doi:10.3390/jcm9030613

Neumann, A. A., Watson, C. M., Noble, J. R., Pickett, H. A., Tam, P. P. L., and Reddel, R. R. (2013). Alternative Lengthening of Telomeres in Normal Mammalian Somatic Cells. Genes Dev. 27 (1), 18-23. doi:10.1101/ gad.205062.112

Paulsen, T., Kumar, P., Koseoglu, M. M., and Dutta, A. (2018). Discoveries of Extrachromosomal Circles of DNA in normal and Tumor Cells. Trends Genet. 34 (4), 270-278. doi:10.1016/j.tig.2017.12.010

Paulsen, T., Malapati, P., Shibata, Y., Wilson, B., Eki, R., Benamar, M., et al. (2021). MicroDNA Levels Are Dependent on MMEJ, Repressed by C-NHEJ Pathway, and Stimulated by DNA Damage. Nucleic Acids Res. 49, 11787-11799. doi:10.1093/nar/gkab984

Paulsen, T., Shibata, Y., Kumar, P., Dillon, L., and Dutta, A. (2019). Small Extrachromosomal Circular DNAs, microDNA, Produce Short Regulatory RNAs that Suppress Gene Expression Independent of Canonical Promoters. Nucleic Acids Res. 47 (9), 4586-4596. doi:10.1093/nar/gkz155

Pavri, R. (2017). R Loops in the Regulation of Antibody Gene Diversification. Genes. 8 (6), 154. doi:10.3390/genes 8060154

Poirier, E. Z., Goic, B., Tomé-Poderti, L., Frangeul, L., Boussier, J., Gausson, V., et al. (2018). Dicer-2-Dependent Generation of Viral DNA from Defective Genomes of RNA Viruses Modulates Antiviral Immunity in Insects. Cell Host \& Microbe. 23 (3), 353-365. doi:10.1016/j.chom.2018.02.001

Prada-Luengo, I., Krogh, A., Maretty, L., and Regenberg, B. (2019). Sensitive Detection of Circular DNAs at Single-Nucleotide Resolution Using Guided Realignment of Partially Aligned Reads. BMC Bioinformatics. 20 (1), 663. doi:10.1186/s12859-019-3160-3

Radloff, R., Bauer, W., and Vinograd, J. (1967). A Dye-Buoyant-Density Method for the Detection and Isolation of Closed Circular Duplex DNA: The Closed Circular DNA in HeLa Cells. Proc. Natl. Acad. Sci. 57 (5), 1514-1521. doi:10.1073/pnas.57.5.1514

Rajkumar, U., Turner, K., Luebeck, J., Deshpande, V., Chandraker, M., Mischel, P., et al. (2019). EcSeg: Semantic Segmentation of Metaphase Images Containing Extrachromosomal DNA. iScience. 21, 428-435. doi:10.1016/j.isci.2019.10.035 
Reon, B. J., and Dutta, A. (2016). Biological Processes Discovered by HighThroughput Sequencing. Am. J. Pathol. 186 (4), 722-732. doi:10.1016/ j.ajpath.2015.10.033

Shibata, Y., Kumar, P., Layer, R., Willcox, S., Gagan, J. R., Griffith, J. D., et al. (2012). Extrachromosomal MicroDNAs and Chromosomal Microdeletions in Normal Tissues. Science. 336 (6077), 82-86. doi:10.1126/science.1213307

Shoura, M. J., Gabdank, I., Hansen, L., Merker, J., Gotlib, J., Levene, S. D., et al. (2017). Intricate and Cell Type-Specific Populations of Endogenous Circular DNA (eccDNA) in Caenorhabditis elegans and Homo sapiens. G3 (Bethesda). 7 (10), 3295-3303. doi:10.1534/g3.117.300141

Sin, S. T. K., Ji, L., Deng, J., Jiang, P., Cheng, S. H., Heung, M. M. S., et al. (2021). Characteristics of Fetal Extrachromosomal Circular DNA in Maternal Plasma: Methylation Status and Clearance. Clin. Chem. 67 (5), 788-796. doi:10.1093/ clinchem/hvaa326

Sin, S. T. K., Jiang, P., Deng, J., Ji, L., Cheng, S. H., Dutta, A., et al. (2020). Identification and Characterization of Extrachromosomal Circular DNA in Maternal Plasma. Proc. Natl. Acad. Sci. USA. 117 (3), 1658-1665. doi:10.1073/ pnas. 1914949117

Sozzi, G., Conte, D., Leon, M., Cirincione, R., Roz, L., Ratcliffe, C., et al. (2003). Quantification of Free Circulating DNA as a Diagnostic Marker in Lung Cancer. J. Clin. Oncol. 21 (21), 3902-3908. doi:10.1200/ JCO.2003.02.006

Sun, Y., Zhang, L., Ho, S. S., Wu, X., and Gu, J. (2016). Lower Mitochondrial DNA Copy Number in Peripheral Blood Leukocytes Increases the Risk of Endometrial Cancer. Mol. Carcinog. 55 (6), 1111-1117. doi:10.1002/ mc. 22373

Sunnerhagen, P., SjÖberg, R.-M., Karlsson, A.-L., Lundh, L., and Bjursell, G. (1986). Molecular Cloning and Characterization of Small Polydisperse Circular DNA from Mouse 3T6 Cells. Nucl. Acids Res. 14 (20), 7823-7838. doi:10.1093/ nar/14.20.7823

Tu, H., Gu, J., Meng, Q. H., Kim, J., Davis, J. W., He, Y., et al. (2015). Mitochondrial DNA Copy Number in Peripheral Blood Leukocytes and the Aggressiveness of Localized Prostate Cancer. Oncotarget. 6 (39), 41988-41996. doi:10.18632/ oncotarget.5889

Turner, K. M., Deshpande, V., Beyter, D., Koga, T., Rusert, J., Lee, C., et al. (2017). Extrachromosomal Oncogene Amplification Drives Tumour Evolution and Genetic Heterogeneity. Nature. 543 (7643), 122-125. doi:10.1038/nature21356 van den Bos, H., Bakker, B., Spierings, D. C. J., Lansdorp, P. M., and Foijer, F. (2018). Single-cell Sequencing to Quantify Genomic Integrity in Cancer. Int. J. Biochem. Cell Biol. 94, 146-150. doi:10.1016/j.biocel.2017.09.016

Van Roy, N., Vandesompele, J., Menten, B., Nilsson, H., De Smet, E., Rocchi, M., et al. (2006). Translocation-Excision-Deletion-Amplification Mechanism Leading to Nonsyntenic Coamplification ofMYC andATBF1. Genes Chromosom. Cancer. 45 (2), 107-117. doi:10.1002/gcc.20272

Wahl, G. M., Robert de Saint Vincent, B., and DeRose, M. L. (1984). Effect of Chromosomal Position on Amplification of Transfected Genes in Animal Cells. Nature. 307 (307), 516-520. doi:10.1038/307516a0

Wang, K., Tian, H., Wang, L., Wang, L., Tan, Y., Zhang, Z., et al. (2021). Deciphering Extrachromosomal Circular DNA in Arabidopsis. Comput. Struct. Biotechnol. J. 19, 1176-1183. doi:10.1016/j.csbj.2021.01.043
Wang, M., Chen, X., Yu, F., Ding, H., Zhang, Y., and Wang, K. (2021). Extrachromosomal Circular DNAs: Origin, Formation and Emerging Function in Cancer. Int. J. Biol. Sci. 17 (4), 1010-1025. doi:10.7150/ijbs.54614

Wang, T., Zhang, H., Zhou, Y., and Shi, J. (2021). Extrachromosomal Circular DNA: A New Potential Role in Cancer Progression. J. Transl. Med. 19 (1), 257. doi:10.1186/s12967-021-02927-x

Wang, Y., Wang, M., Djekidel, M. N., Chen, H., Liu, D., Alt, F. W., et al. (2021). EccDNAs Are Apoptotic Products with High Innate Immunostimulatory Activity. Nature. 599, 308-314. doi:10.1038/s41586-021-04009-w

Wiedemann, E.-M., Peycheva, M., and Pavri, R. (2016). DNA Replication Origins in Immunoglobulin Switch Regions Regulate Class Switch Recombination in an R-loop-dependent Manner. Cell Rep. 17 (11), 2927-2942. doi:10.1016/ j.celrep.2016.11.041

Wu, S., Turner, K. M., and Nguyen, N. (2019). Circular ecDNA Promotes Accessible Chromatin and High Oncogene Expression. Nature. 575, 699. doi:10.1038/s41586-019-1763-5

Yamagishi, H., Kunisada, T., and Takeda, T. (1985). Amplification of Extrachromosomal Small Circular DNAs in a Murine Model of Accelerated Senescence. A Brief Note. Mech. Ageing Development. 29 (1), 101-103. doi:10.1016/0047-6374(85)90051-x

Yerlici, V. T., Lu, M. W., Hoge, C. R., Miller, R. V., Neme, R., Khurana, J. S., et al. (2019). Programmed Genome Rearrangements in Oxytricha Produce Transcriptionally Active Extrachromosomal Circular DNA. Nucleic Acids Res. 47 (18), 9741-9760. doi:10.1093/nar/gkz725

Zhou, W., Zhu, M., Gui, M., Huang, L., Long, Z., Wang, L., et al. (2014). Peripheral Blood Mitochondrial DNA Copy Number Is Associated with Prostate Cancer Risk and Tumor burden. PLoS One. 9 (10), e109470. doi:10.1371/ journal.pone.0109470

Zhu, Y., Gujar, A. D., Wong, C.-H., Tjong, H., Ngan, C. Y., Gong, L., et al. (2021). Oncogenic Extrachromosomal DNA Functions as mobile Enhancers to Globally Amplify Chromosomal Transcription. Cancer Cell. 39, 694-707. doi:10.1016/j.ccell.2021.03.006

Conflict of Interest: The authors declare that the research was conducted in the absence of any commercial or financial relationships that could be construed as a potential conflict of interest.

Publisher's Note: All claims expressed in this article are solely those of the authors and do not necessarily represent those of their affiliated organizations, or those of the publisher, the editors, and the reviewers. Any product that may be evaluated in this article, or claim that may be made by its manufacturer, is not guaranteed or endorsed by the publisher.

Copyright (c) 2022 Zuo, Yi, Wang, Li, Zhou, Peng, Zhou, Yang and He. This is an open-access article distributed under the terms of the Creative Commons Attribution License (CC BY). The use, distribution or reproduction in other forums is permitted, provided the original author(s) and the copyright owner(s) are credited and that the original publication in this journal is cited, in accordance with accepted academic practice. No use, distribution or reproduction is permitted which does not comply with these terms. 